\title{
The Influence of Rotating Direction on the Tribological Behavior of Grey Cast Iron with Curve Distributed Pit Textured Surface
}

\author{
Shaoni Sun, ${ }^{1}$ Liyang Xie, ${ }^{1}$ Peter Kelly, ${ }^{2}$ Risheng Long, ${ }^{3,4}$ Manhong Li, ${ }^{5}$ and Jiling Feng ${ }^{6}$ \\ ${ }^{1}$ School of Mechanical Engineering and Automation, Northeastern University, Shenyang, Liaoning 110819, China \\ ${ }^{2}$ Surface Engineering Group, Manchester Metropolitan University, Manchester M1 5GD, UK \\ ${ }^{3}$ College of Mechanical Engineering, Taiyuan University of Technology, Taiyuan, Shanxi 030024, China \\ ${ }^{4}$ Shanxi Key Laboratory of Fully Mechanized Coal Mining Equipment, Taiyuan, Shanxi 030024, China \\ ${ }^{5}$ Changsha Research Institute of Mining and Metallurgy Co., Ltd., Changsha, Hunan 410012, China \\ ${ }^{6}$ School of Engineering, Division of Mechanical Engineering, Manchester Metropolitan University, Manchester M1 5GD, UK
}

Correspondence should be addressed to Liyang Xie; lyxie@me.neu.edu.cn and Peter Kelly; peter.kelly@mmu.ac.uk

Received 11 May 2017; Revised 3 August 2017; Accepted 13 August 2017; Published 26 September 2017

Academic Editor: Denis Benasciutti

Copyright (C) 2017 Shaoni Sun et al. This is an open access article distributed under the Creative Commons Attribution License, which permits unrestricted use, distribution, and reproduction in any medium, provided the original work is properly cited.

\begin{abstract}
To investigate the influence of surface features, in the form of pits, on the wear resistance of grey cast iron (GCI), a finite element model of the pin-on-disc friction system, with pits distributed in a curved radial direction, was developed using APDL programming and the tribological behavior of textured surfaces was studied. The influence of relative rotation direction between the disc and the pin on the thermal behavior of the friction system under dry wear conditions was researched. GCI and C30E steel samples with pit textured surfaces were manufactured using laser marking equipment and tested using a tribology wear testing rig. The mass losses were measured and the worn surfaces were characterized. The influence of different rotation directions on the tribological behavior of the pit textured surfaces was also investigated. The simulation and test results revealed that rotation direction was a crucial parameter in determining the tribological behavior of surfaces with these features, regardless of the material. Under the conditions tested, when the pin rotated anticlockwise, the samples showed better friction and wear behavior than when the pin rotated clockwise. These results can provide important guidance for the optimization of the design of heavy-load brake systems and other similar applications.
\end{abstract}

\section{Introduction}

Owing to its low cost, good damping property, fine castability, and machinability, grey cast iron (GCI) is widely used in many fields, such as machine tools (base and guideways), vehicle engines (shell and cylinders), reduction gearboxes (shell), and brake discs of trains [1]. The wear resistance of GCI is a very important index in choosing this material for these applications and directly affects the service life of the whole system, especially under dry wear condition [2]. Therefore, it is of great significance to investigate feasible means of improving the wear resistance of GCI parts under dry sliding conditions.

Surface texturing is one of the main methods to control friction, improve the reliability, and prolong the service life of mechanical parts in sliding wear applications [3-8]. The surface patterns, such as "pit or spot," "groove or striation," and "grid or lattice," may be inspired by the natural flora and fauna $[9,10]$ or regularly distributed features [11-13], isotropic or anisotropic [14-19]. "Stick-slip" friction phenomena can be observed during sliding, depending on the surface topological pattern. To improve the wear resistance of mechanical parts, great efforts have been theoretically and experimentally made to investigate the orientation effects between frictional motion direction and surface texture units or patterns, on the tribological behavior under different lubrication conditions [18-23]. Yuan et al. [20] studied the orientation effects of grooves on the friction performance. The results indicated that grooves perpendicular or parallel to the sliding direction had a strong impact on the friction performance of 


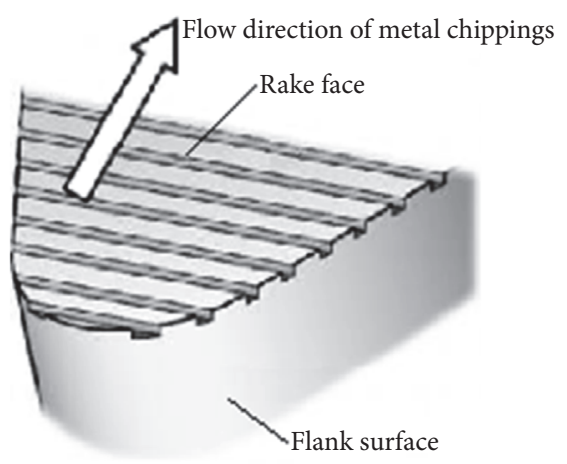

(a) Horizontal

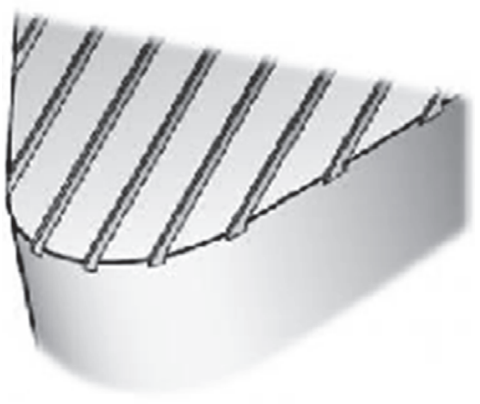

(b) Vertical

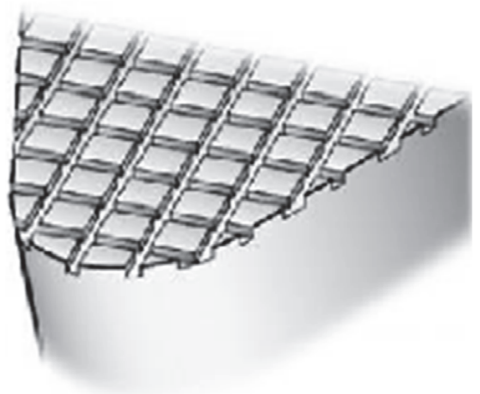

(c) Rectangle

FIGURE 1: Different grooves on the cutting tools processed by laser machining [23].

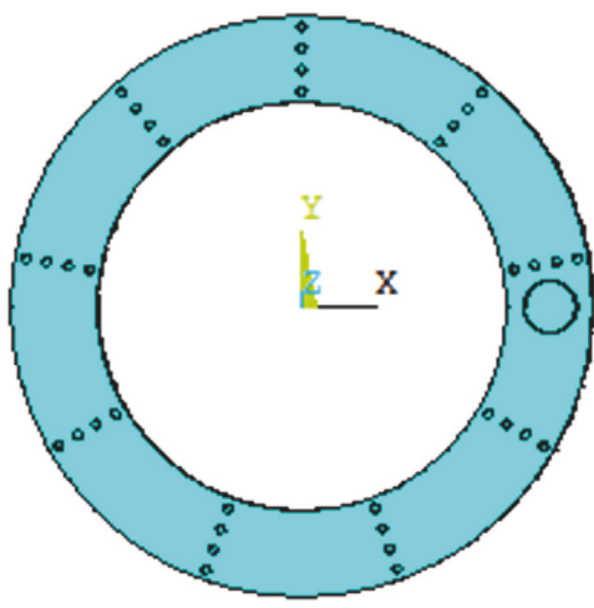

(a) LDRD

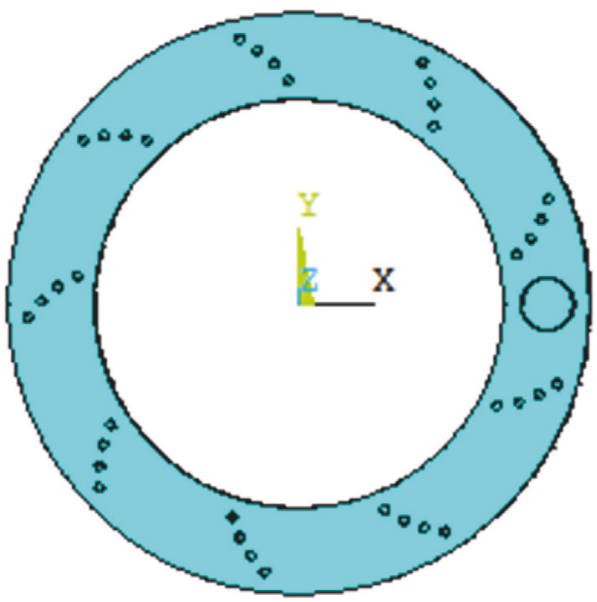

(b) CDRD

FIGURE 2: Diagram of LDRD and CDRD surfaces.

sliding surfaces, and the merits of perpendicular or parallel orientation might swap under different contact conditions. Costa and Hutchings [21] fabricated regularly distributed micro-dimples and grooves on the surface of moulds through photochemical technology and found that when a high density (about 25\%) of grooves was perpendicular to the direction of motion, the friction coefficient could be reduced by a great degree under oil lubrication. If the grooves were parallel to the motion direction, the friction coefficient increased, with poor wear resistance. Pettersson and Jacobson [22] researched the effect of the angle of the groove on the tribological behavior using a ball-on-disc friction testing machine under boundary lubrication conditions. The results showed that when the grooves were parallel with the direction of movement, the friction force and wear loss were much larger than when the grooves were perpendicular to the direction of motion. The Japanese Toyama University and Toyama Industrial Technology Center [23] processed regularly distributed micro- and nanogrooves on the rake surface of cutting tools by femtosecond laser machining (see Figure 1). The experimental data from actual machining processes of aluminum alloys using these tools revealed that surface texture could effectively reduce the cutting force by decreasing the friction force on the flank face of tools, especially when the grooves were perpendicular to the flow direction of the metal chippings. Therefore, the direction of motion is of great significance for textured surfaces, which will induce different tribological behavior in different directions. The above findings are mainly from experimental studies, and there are few publications studying the friction and wear behavior of GCI parts with curve distributed pit textured surfaces, especially through numerical simulation methods.

Based on previous research on the thermal behavior of a pin-on-disc friction system, where pits were distributed in the linear radial direction (LDRD) on the upper surface of the disc (see Figure 2(a)) [24], a new finite element model with curve distributed pits in the radial direction (CDRD) (see Figure 2(b)) was established through APDL (ANSYS parametric design language) programming in this work. The influence of the rotation direction of the pin on the thermal behavior of the pin-on-disc system was investigated. In parallel, wear tests of GCI and C30E steel specimens with pit surfaces prepared by a laser marking machine were 


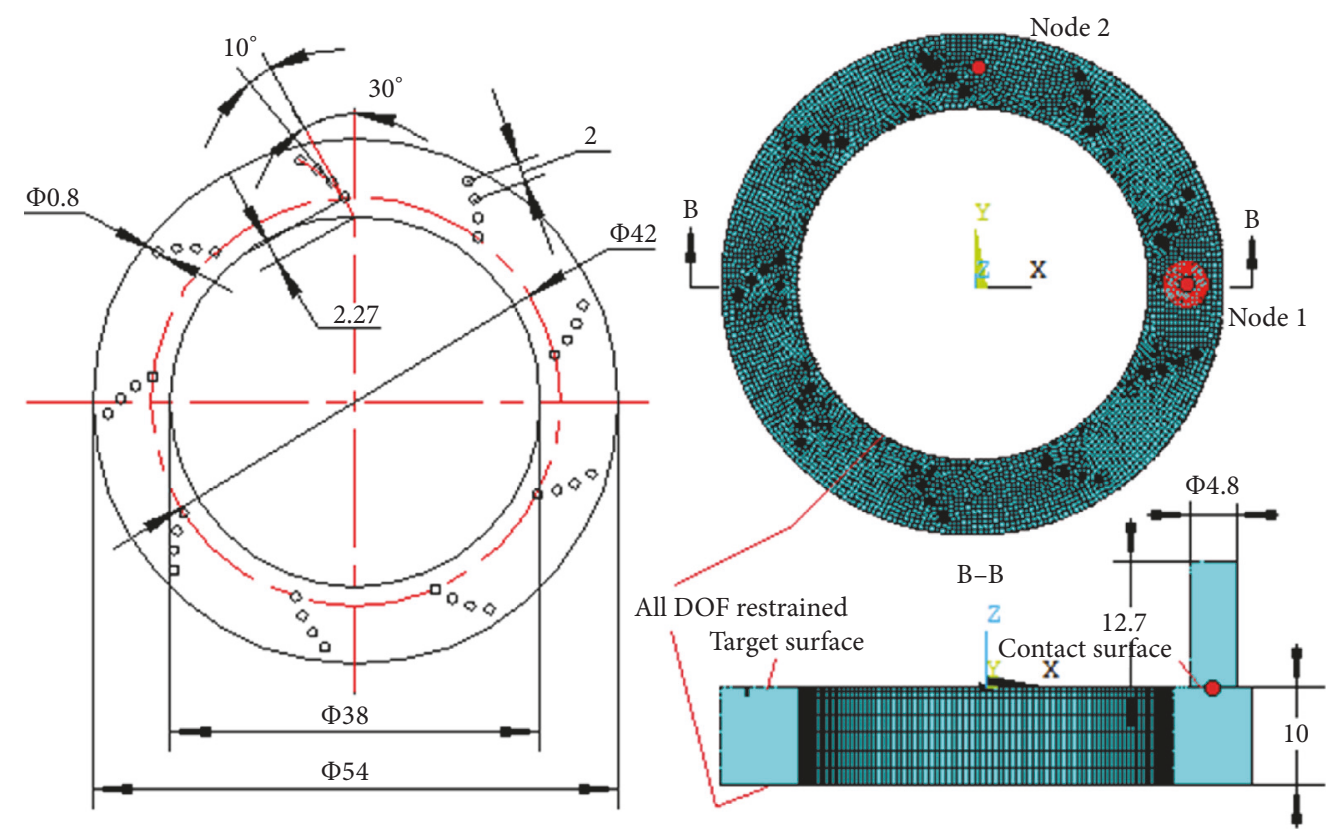

FIGURE 3: Sketch of the pit textured pin-on-disc friction system.

TABLE 1: Thermal physical properties of GCI and H13 tool steel.

\begin{tabular}{lcc}
\hline Thermal physical parameter & GCI & H13 tool steel \\
\hline Thermal conductivity, $K_{e}, \mathrm{~W} /(\mathrm{m} \cdot \mathrm{K})$ & 48 & 32.2 \\
Density, $\rho, \mathrm{kg} / \mathrm{m}^{3}$ & 7200 & 7800 \\
Specific heat capacity, $c, \mathrm{~J} /(\mathrm{kg} \cdot \mathrm{K})$ & 480 & 460 \\
Thermal expansion coefficient, $\alpha, \times 10^{-5} \mathrm{~K}^{-1}$ & 1.2 & 0.91 \\
Elastic modulus, $E, \mathrm{Gpa}$ & 120 & 210 \\
Poisson ratio, $v$ & 0.25 & 0.30
\end{tabular}

carried out using a tribology wear testing rig at Manchester Metropolitan University (MMU). The friction coefficient curves and mass losses of the samples were obtained, and the morphologies of the worn surfaces were characterized by stereo optical microscopy.

\section{Design of Pit Textured Surfaces}

The pit textured discs, fabricated from GCI, were dimensioned for the pin-on-disc wear tester, that is, outer diameter, $\Phi 54 \mathrm{~mm}$, and inner diameter, $\Phi 38 \mathrm{~mm}$, with a thickness of $10 \mathrm{~mm}$ (see Figure 3). The size of the pin was $\Phi 4.8 \mathrm{~mm} \times$ $12.7 \mathrm{~mm}$, manufactured from $\mathrm{H} 13$ tool steel. The thermal physical properties of GCI and H13 tool steel are both listed in Table 1.

The design of the textured surfaces is the basis of this work. As shown in Figure 3, the main parameters of the pit textured surfaces include diameter of pit (DAOP), depth of pit (DPOP), radial spacing of adjacent pits (RSAP), number of pit sets (NPS), initial deflection angle of the first pit (IDAFP), and deflection angle of adjacent pits (DAAP). The optimal surface texture parameters obtained in previous research were used, that is, the DAOP, $0.8 \mathrm{~mm}$, and the DPOP, $1.0 \mathrm{~mm}$ [24]. Other parameters were set to RSAP, $2 \mathrm{~mm}$; the IDAFP, $30^{\circ}$; and DAAP, $10^{\circ}$. Evidently, as shown in the figure, there are nine groups of uniformly distributed pits in total, and each set includes four pits. The diameter of the circle, which passes through the center of the innermost pit in each group is $\Phi 42 \mathrm{~mm}$. The positions of the other pits are determined by the RSAP, IDAFP, and DAAP values.

\section{Simulation and Results Discussion}

Friction and wear is a thermodynamically nonequilibrium process. The drastic variations of temperature may cause phase transitions of metal in the near surface region and further affect the tribological behavior. Therefore, to determine the thermal behavior of the pin-on-disc friction system in different rotation directions, in relation to the pin locations, and further study the influence of this parameter on the tribological properties of the pit textured surfaces, the following work was undertaken through the APDL programming.

3.1. Thermodynamics Theory and Conditions. For certain conditions, the heat conduction during friction processes can be described by the classical heat conduction equation. If we set $k_{e}=k_{e 1}+k_{e 2}, c=c_{1}+c_{2}$, and $\rho=\rho_{1}+\rho_{2}$ and take heat convection and heat radiation into account, the classical heat conduction equation can be expressed as the following in the Cartesian coordinate system:

$$
\begin{gathered}
k_{e}\left(\frac{\partial^{2} T}{\partial x^{2}}+\frac{\partial^{2} T}{\partial y^{2}}+\frac{\partial^{2} T}{\partial z^{2}}\right)+q+h\left(T-T_{h}\right) \\
+\sigma \varepsilon\left(T^{4}-T_{h}^{4}\right)=\rho \cdot c \frac{\partial T}{\partial t}
\end{gathered}
$$

where $k_{e}$ is the thermal conductivity of friction system, $c$ is the specific heat capacity of friction system, $q$ is the heat flux 
through the contact area, $\rho$ is the density of friction system, $h$ is the convective heat transfer coefficient, $T_{h}$ is the ambient temperature, $\sigma$ is the radiation constant of a blackbody, and $\varepsilon$ is the radiation rate of the actual object.

To solve (1), the initial condition and boundary conditions are necessary. In this work, the initial condition is the original temperature of the pin-on-disc system at the beginning of the simulation, equal to $20^{\circ} \mathrm{C}$. The boundary condition is the heat exchange condition between the outer surface of the pin-on-disc system and its surrounding environment. Generally, the boundary conditions can be classified into the following four kinds.

The first boundary condition is the boundary temperature of objects, a known constant or function of position and time. It can be given as

$$
\begin{aligned}
\left.T\right|_{S} & =T_{S} \\
\text { or }\left.T\right|_{S} & =T_{S}(x, y, z, t),
\end{aligned}
$$

where $S$ is the boundary of the pin-on-disc friction system.

The second boundary condition refers to the heat flux, $q_{w}$, at the boundary of the objects, which is a known constant or also a function of position and time. For pin-on-disc friction systems, it can be expressed as

$$
\begin{aligned}
-\left.k(T) \frac{\partial T}{\partial \vec{n}}\right|_{S} & =q_{w} \\
\text { or }-\left.k(T) \frac{\partial T}{\partial \vec{n}}\right|_{S} & =q_{w}(x, y, z, t) \\
q_{w}(x, y, z, t) & =\eta \cdot \mu \cdot P \cdot v(x, y, z, t),
\end{aligned}
$$

where $q_{w}$ is the heat flux through the contact surface of the pin-on-disc system, a known constant; $q_{w}(x, y, z, t)$ is the expression of input heat flux, a function of position and time; $\eta$ is the conversion efficiency, $0.85 ; \mu$ is the friction coefficient of the system, $0.38 ; P$ is the normal pressure of the pin; $v(x, y, z, t)$ is the relative linear velocity between disc and pin. Meanwhile, the lower surface of the disc can be regarded as being in the adiabatic state, whose boundary condition can be written as

$$
-k_{e} \frac{\partial T}{\partial z}=0
$$

The third boundary condition is the heat transfer coefficient of the medium in the surrounding environment. The most commonly used one is the mixed convection and radiation heat transfer boundary condition, which can be displayed as

$$
-\left.k(T) \frac{\partial T}{\partial \vec{n}}\right|_{S}=h\left(T_{S}-T_{e}\right)+\sigma \varepsilon\left(T_{S}^{4}-T_{e}^{4}\right),
$$

where $\sigma$ is the Stefan-Boltzmann constant; $\varepsilon$ is the radiation rate of the pin-on-disc system.

The fourth boundary condition is the heat conduction between the disc and the pin and can be expressed as

$$
-\left.\lambda_{n} \frac{\partial T}{\partial n}\right|_{S}=\beta\left(T_{S}-T_{e}\right),
$$

where $T_{S}$ is the temperature of the disc or pin, $T_{e}$ is the temperature of the environment, and $\beta$ is the heat transfer coefficient between the disc and the pin.

For pin-on-disc friction system, the temperature distribution in the disc or the pin is very uneven during dry wear processes. As a result, a huge temperature gradient exists in the disc, which further induces high thermal stresses. Thus, the thermal strain is composed of two parts: one is caused by temperature variation, and the other is caused by stress.

3.2. Modelling and Setting. The contact surface between the pit textured disc and the pin was set as a friction pair, wherein the upper surface of the disc was the "target surface," while the lower surface of the pin was the "contact surface" (see Figure 3). During the simulation process, both faces were always kept in contact with each other under a vertical force of $70 \mathrm{~N}$, equivalent to a pressure of approximately 0.98 MPa. As shown in Figure 3, to intuitively display the relative positions of the disc and pin, the disc was constricted in all DOFs based on the equivalent principle in the whole simulation. Conversely, an angular velocity of $25 \mathrm{rad} / \mathrm{s}$ (about $200 \mathrm{RPM}$ ) was applied to the pin, which could rotate anticlockwise or clockwise, respectively. In consideration of the performance of the computer used, the duration of the simulation was set to $0.5 \mathrm{~s}$ (100 substeps, i.e., 0.005 seconds per substep); thus it is only the heat conduction of the immediate friction interface that was considered during the simulation.

The element type used in this work includes SOLID226, TARGE170, CONTA174, and MPC184. The number of elements is 40962. To easily understand and compare the obtained data, as shown in Figure 3, two typical nodes, node 1 and node 2, were selected to demonstrate the thermal behavior of the pit textured surface in different rotation directions. Specifically, node 1 was on the upper surface of the disc, located at the middle of the initial contact face between the disc and the pin. Node 2 was also on the upper surface of the disc but rotated by 90 degrees anticlockwise.

\subsection{Results and Discussion}

3.3.1. Temperature Behavior of the Textured Surfaces. Figure 4 shows the temperature contours of the pin-on-disc system for different rotation directions at the end of the simulation. As demonstrated in the figure, when the pin rotated anticlockwise, the heat affected region of the textured disc was larger than the affected area as the pin rotated in the contradirection. Furthermore, there was an evident uneven temperature phenomenon, which appeared on the pit textured surface that might be caused by the former mentioned "stick-slip friction," whether the pin rotated in the clockwise or anticlockwise direction. This phenomenon could be called "temperature crawling" and was more obvious when the pin rotated clockwise.

Figure 5 displays the temperature-time curves of node 1 and node 2 when the pin rotates in different directions. As shown in Figure 5(a), the temperature of node 1 gradually increased at the outset of the simulation, whether the pin rotated clockwise or anticlockwise. Both of the two curves were similar and had three obvious peaks at the $0.13 \mathrm{~s}$, 


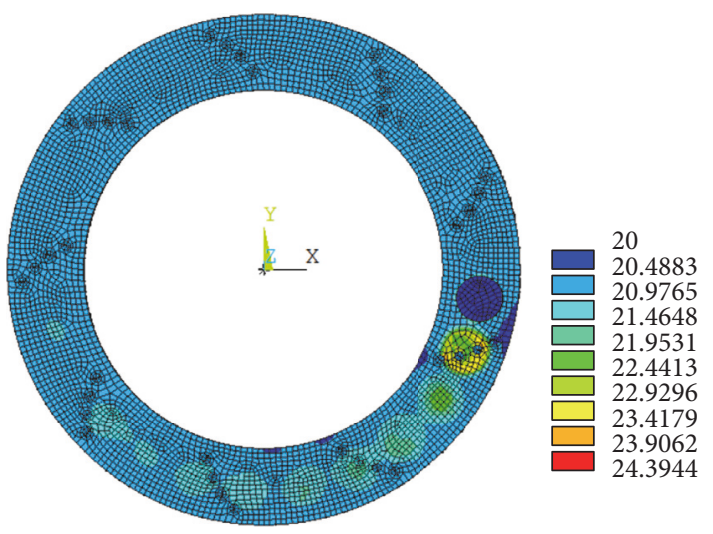

(a) Pin rotated anticlockwise

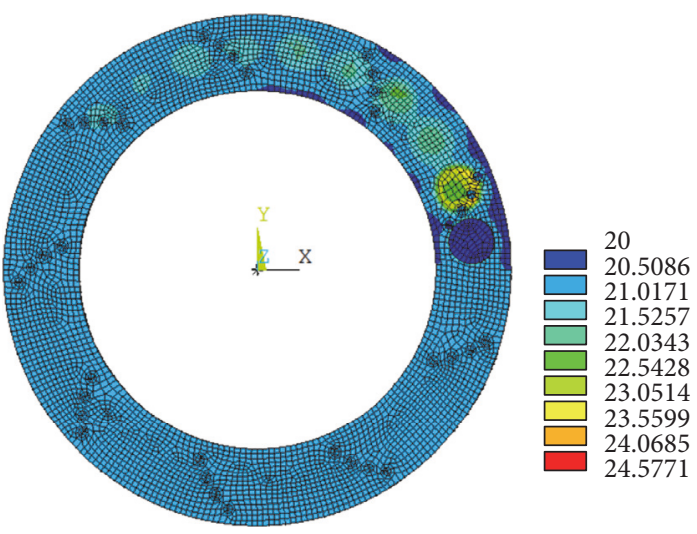

(b) Pin rotated clockwise

FIGURE 4: Temperature contours of pit textured surfaces after $0.5 \mathrm{~s}$ in different rotation directions.

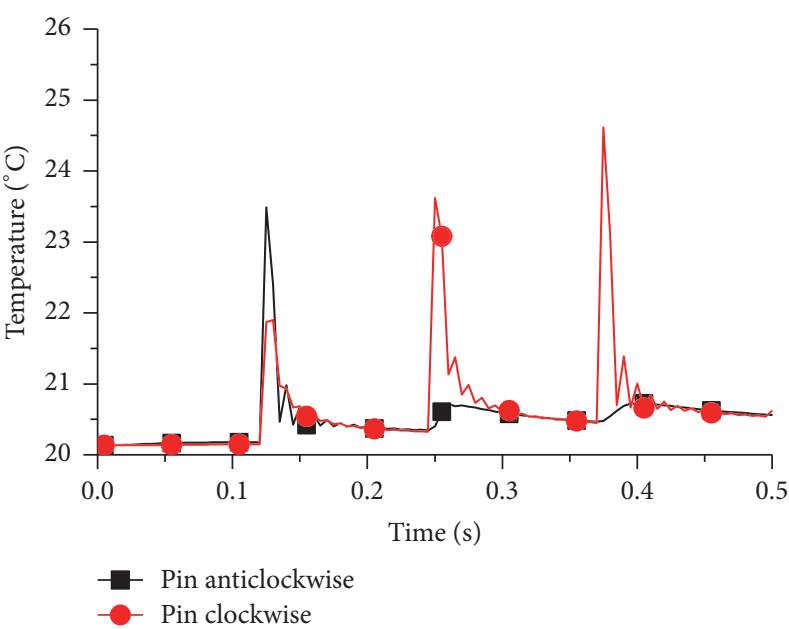

(a) Node 1

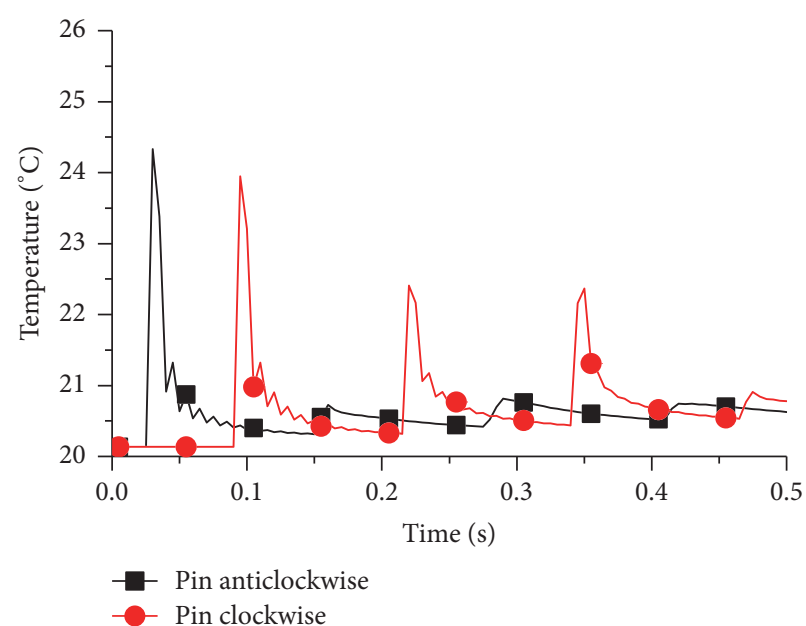

(b) Node 2

FIgURE 5: Temperature-time curves of node 1 and node 2 in different rotation directions.

$0.25 \mathrm{~s}$, and $0.38 \mathrm{~s}$, respectively, corresponding to each moment where the pin passed over the node. Clearly, when the pin rotated anticlockwise, the average temperature of node 1 was lower than the temperature as the pin rotated in the clockwise direction, except for the first peak at $0.13 \mathrm{~s}$. The temperatures of node 1 in the two rotation directions at $0.25 \mathrm{~s}$ were $20.4^{\circ} \mathrm{C}$ and $23.6^{\circ} \mathrm{C}$, respectively, which equated to a temperature difference of $15.7 \%$. The temperature difference of node 1 at $0.38 \mathrm{~s}$ was about $20.1 \%$, the highest of the three peaks. For node 2, the two temperature-time curves, when the pin rotated clockwise and anticlockwise, also exhibited slow upward trends, with three peaks (see Figure 5(b)). It is evident that when the pin rotated in the anticlockwise direction, the average temperature of node 2 was also lower than the temperature when the pin rotated in the contradirection.

By comparing Figures 4 and 5, whether from the curves or contours, it is apparent that when the pin rotated anticlockwise, the pit textured surface exhibited slightly lower temperatures than when the pin rotated clockwise during the whole simulation, indicating a lower rate of temperature rise.
Based on the relationship between temperature and friction and wear performance, under the same loading parameters and duration of simulation, the textured surface with the lower temperature rise would also be expected to show a lower friction coefficient and better wear resistance.

3.3.2. Thermal Stress Behavior of the Textured Surface. Figure 6 displays the von Mises stress contours of the pit textured surfaces in different rotation directions at the end of the simulation. As shown in the figure, for both rotation directions, the high von Mises stress region was always located at the inner ring of the disc, because of the displacement constraint in all DOFs during the simulation, and extended towards the pit groups. Meanwhile, the von Mises stress around the pits group was lower than the stresses in the smooth area without pits (see the enlarged view of Figure 7). The highest von Mises stress region of the textured surface was the area that the pin had just passed over. By analyzing the simulation results, it is obvious that (1) the pit textured structure has an adjusting effect on the stress distribution of the whole disc and (2) the relative rotation direction between 


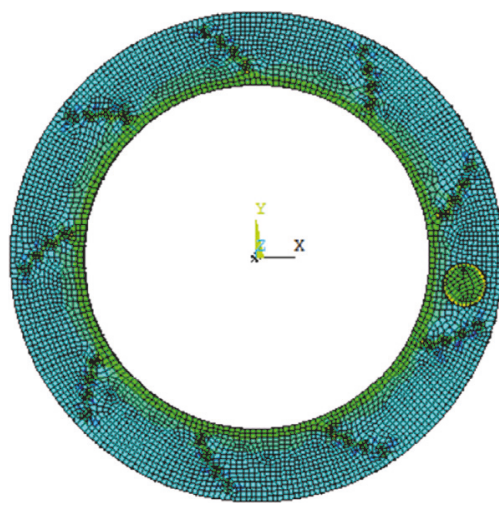

(a) Pin rotated anticlockwise

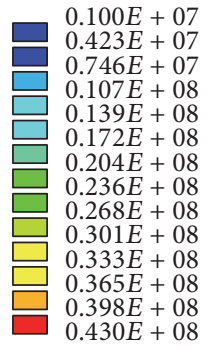

IGURE 6: The von Mises stress contours of pit textured surfaces in different rotation directions.

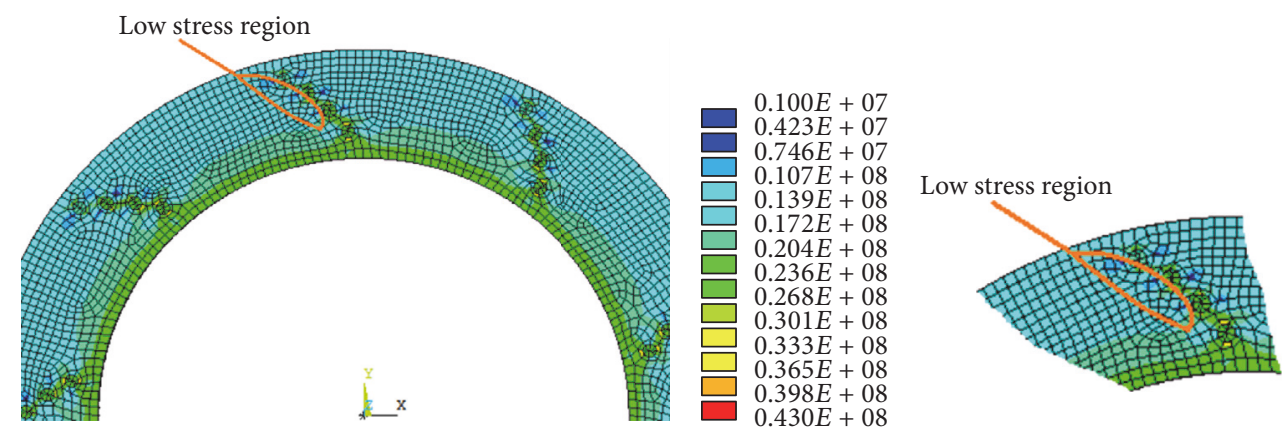

FIGURE 7: Enlarged contour of low von Mises stress region around the pit set.

disc and pin can also alter the thermal stress behavior of the textured surface.

Figure 8(a) shows the von Mises stress-time curves of node 1 when the pin rotated in two different directions. Obviously, both of the curves fluctuated periodically, and the von Mises stress decreased firstly and then increased, showing an upward trend on the whole. In general, when the pin rotated anticlockwise, the average von Mises stress of node 1 was smaller than the stress when the pin rotated in the contradirection. Figure 8(b) demonstrates the von Mises stress-time curves of node 2 in different rotation directions. The two curves showed similar shapes, with the same downward trends and fixed time-lag. Evidently, when the pin rotated anticlockwise, the von Mises stress variation of node 2 and its average value were all less than the stresses when the pin rotated clockwise.

By comparing temperature and von Mises stress data in Figures $4-8$, it is evident that the rotating direction of pin has an effect on the thermal behavior of pit textured surfaces. The temperature and stress of the contact surface can directly affect the friction properties of the textured surface, change the friction coefficient during friction process, and finally induce variations in tribological properties of the whole pinon-disc system.

\section{Wear Test and Results}

To verify the results of the simulations, a group of GCI samples were fabricated by a laser machining system

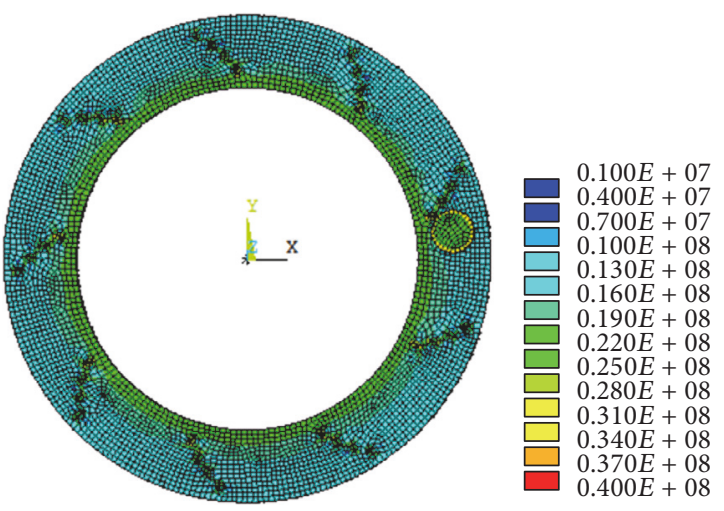

(b) Pin rotated clockwise
$0.400 E+08$ 


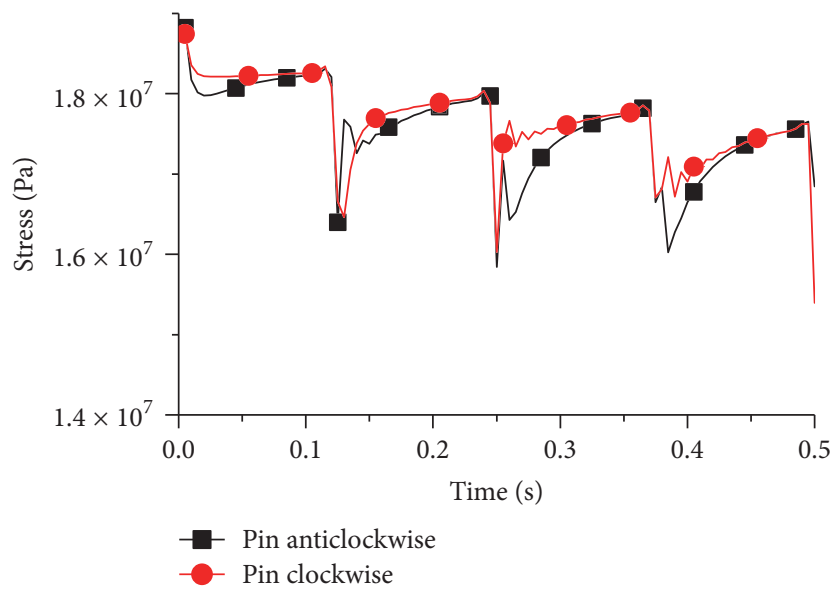

(a) Node 1

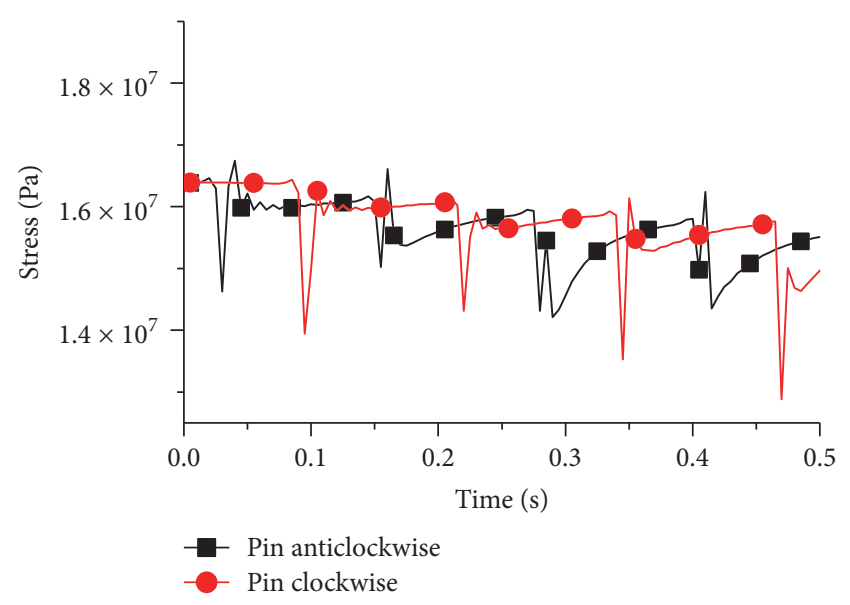

(b) Node 2

FIgURE 8: The von Mises stress-time curves of node 1 and node 2 in different rotation directions.

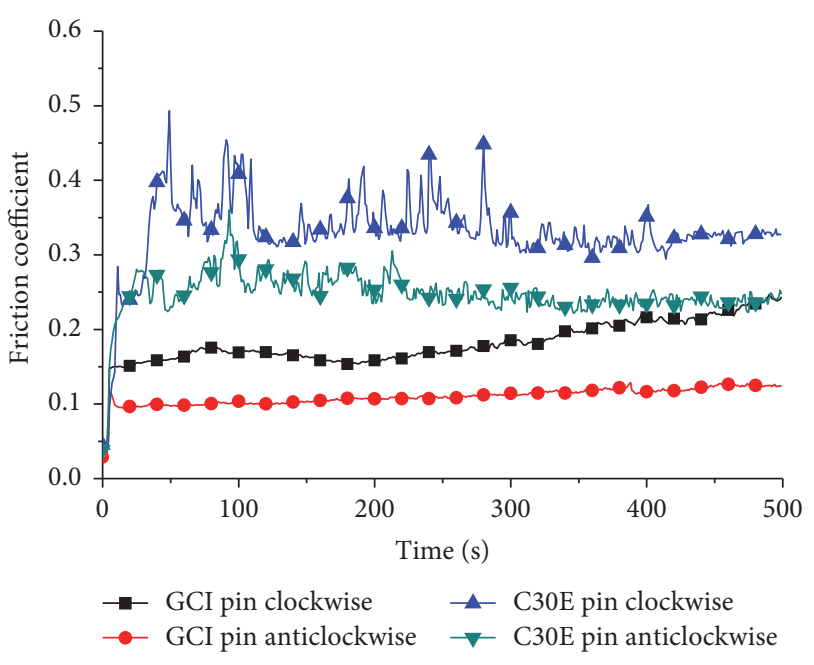

FIGURE 9: Friction coefficient curves of GCI and C30E steel specimens in different rotating directions.

C30E steel samples. For both kinds of materials, the friction responses in different rotation directions were also different. When the pin rotated anticlockwise, the friction coefficient curves were more stable and the friction coefficient values were lower than those observed as the pin rotated in the contradirection.

The worn surfaces of GCI and C30E steel samples as the pin rotated in different directions are shown in Figure 10. The following was found: (1) Under the same friction testing condition, the worn surfaces of samples when the pin rotated in an anticlockwise or clockwise direction were quite different, regardless of the sample materials. (2) When the pin rotated anticlockwise, for the GCI samples, there were only a few visible wear tracks on the worn surface, and the tracks were independent and shallow (see Figure 10(a)). As the pin rotated in the opposite direction, the worn area of the pit textured specimen was much wider and deeper, and the wear tracks became contiguous (see Figure 10(b)). (3) For the
C30E steel samples, there were serious viscous phenomena on the worn surfaces, whether the pin rotated anticlockwise (see Figure 10(c)) or clockwise (see Figure 10(d)). Meanwhile, a continuous sharp noise and juddering accompanied the whole testing process. (4) By comparing Figures 10(c) and $10(\mathrm{~d})$, when the pin rotated clockwise, there were some pieces of debris on the edge of the worn surface after wear testing, and the surface morphology was uneven with many corrugations. This was reduced as the pin rotated in the anticlockwise direction.

Table 2 displays the mass loss of the pit textured samples (GCI and C30E) after wear testing in different rotation directions. It is evident that (1) the mass losses of the $\mathrm{C} 30 \mathrm{E}$ steel specimens were much greater than the losses of GCI samples, regardless of rotation direction, and (2) for both kinds of disc materials, when the pin rotated anticlockwise, the mass loss of the sample was much less than when the pin rotated clockwise. Therefore, the rotation direction of the pin is a significant factor for the wear resistance and tribological behavior of pit textured surfaces, which is also consistent with the former simulation results. Under the loading conditions of this work, with the curved design of the pit layout, the best results were observed when the pin rotated in an anticlockwise direction relative to the surface of the disc.

\section{Discussion}

For the pin-on-disc friction system with curve distributed pit textured surfaces, there is an orientation effect, whether the rotation direction of the pin across the disc is anticlockwise or clockwise. As shown in Figure 11(a), when the pin rotated anticlockwise, which is equivalent to the disc rotating clockwise (i.e., pin kept motionless), the following was shown clearly: (1) The innermost pit (or leading pit) would be the first one to be covered by the pin, followed in succession by the adjacent pits. This successive passage over the pits was found to be beneficial for the heat transfer process, including radiation and convection. As a result, the temperature of the pit textured surface was slightly lower, which was consistent 


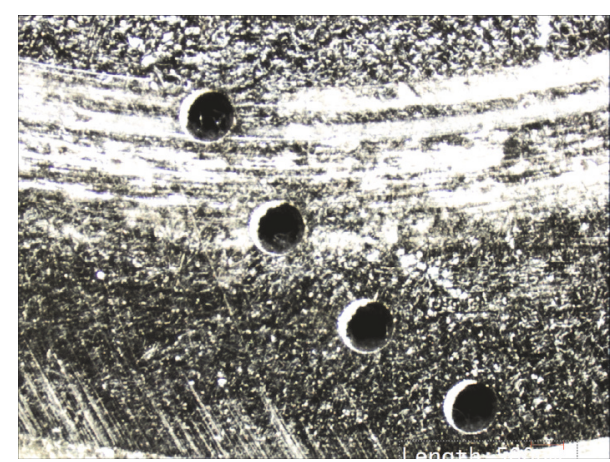

(a) GCI pin anticlockwise

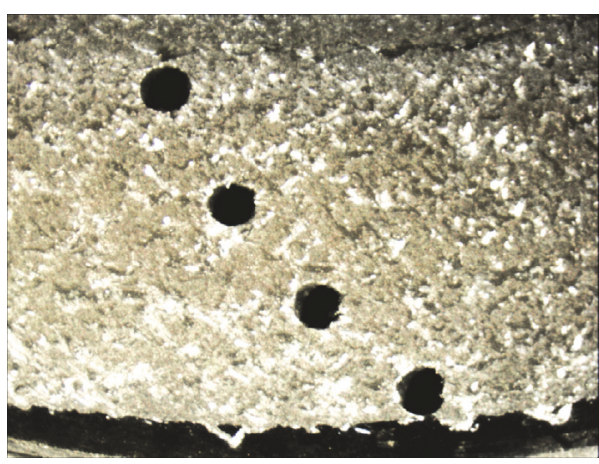

(c) C30E pin anticlockwise

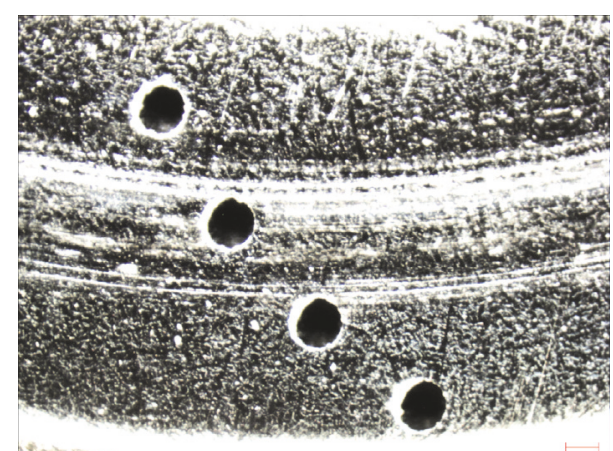

(b) GCI pin clockwise

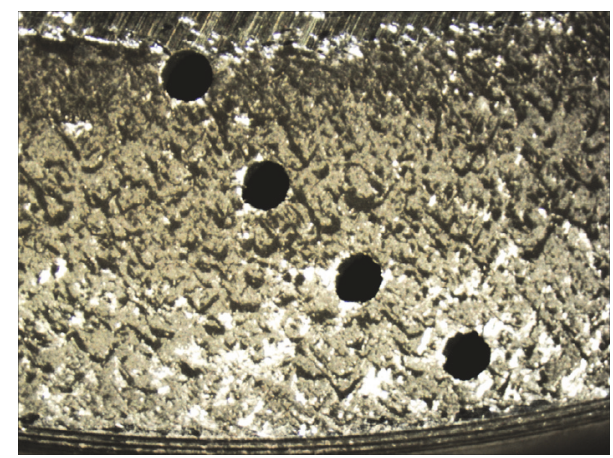

(d) C30E pin clockwise

FIGURE 10: Worn surfaces of GCI and C30E steel samples after wear testing against EN1A counterfaces in different rotation directions.

TABLE 2: Wear loss of GCI and C30E steel samples in different rotation directions.

\begin{tabular}{lccc}
\hline Sample name & Average mass before wear test $(\mathrm{g})$ & Average mass after wear test $(\mathrm{g})$ & Average mass loss $(\mathrm{g})$ \\
\hline GCI pin anticlockwise & 80.71227 & 80.71190 & 0.00037 \\
GCI pin clockwise & 80.19157 & 80.18953 & 0.00203 \\
C30E pin anticlockwise & 88.96153 & 88.91047 & 0.05107 \\
C30E pin clockwise & 87.85777 & 87.70907 & 0.14870 \\
\hline
\end{tabular}

with the slow variation speed of the former temperature behavior analysis. (2) The metal debris in front of the pin tended to be shifted from the inside to the outside of the disc (see the arrows in Figure 11(a)), under the push and squeeze of pin. At this point, the pits would act as a trap, to provide a sink for debris particles to sit in, and reduce the associated additional friction of debris in the contact zone. Under the combined effects of the movement of the pin and centrifugal force, the wear debris could be rapidly removed, which is significant for restricting the friction of the contact surface.

Conversely, as displayed in Figure 11(b), when the pin rotated clockwise, equivalent to the disc rotating in an anticlockwise direction (pin kept motionless), the following results can be obtained: (1) All pits (whether leading pit or trailing pit) were almost passed over and covered by the pin simultaneously. It was disadvantageous for the heat transfer process and would cause a sudden rise in temperature, corresponding to the peaks observed in Figure 5. (2) The metal debris in front of the pin was prone to moving from the outside to the inside of the disc, under the action of the pin (see the arrows in Figure 11(b)). However, the centrifugal force would force wear debris to move along the radial or involute direction, depending on the rotating speed. Finally, the combined effects of the pin as well as centrifugal force would impede or slow the rapid removal of debris, causing a "second grinding" phenomenon on the contact surface, and worsen the tribological behavior of the pit textured surface.

In the practical application of vehicle open brake systems with pits, there are two common installation methods for the brake discs, corresponding to the anticlockwise direction and clockwise direction studied in this work. If a disc was mounted as in Figure 11(a), the wear resistance and durability of the brake disc would be enhanced by rapid removal of the brake debris and relatively lower surface temperatures during the braking process. However, if the brake disc was installed as shown in Figure 11(b), the brake system may provide a better braking performance, compared with the former installation method, but with high wear loss ratio and shorter service life. It is also consistent with the former results; that is, the anticlockwise rotation direction of the pin can provide better wear resistance than the clockwise rotation direction, under the conditions of this study. For the wet braking system, 


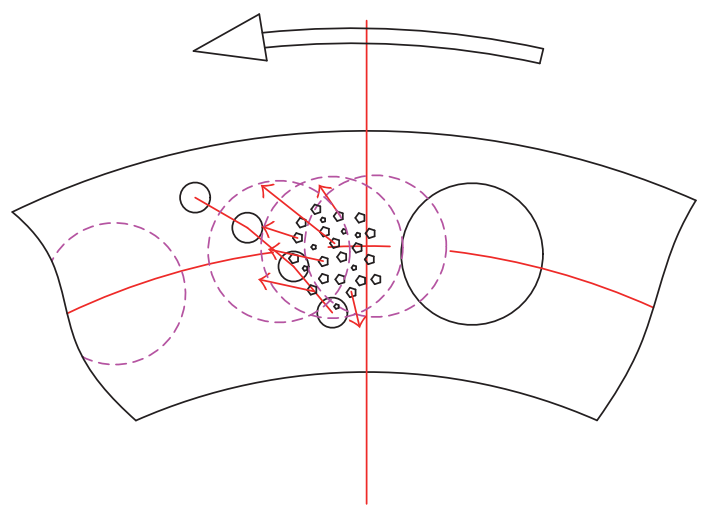

(a) Pin rotated anticlockwise

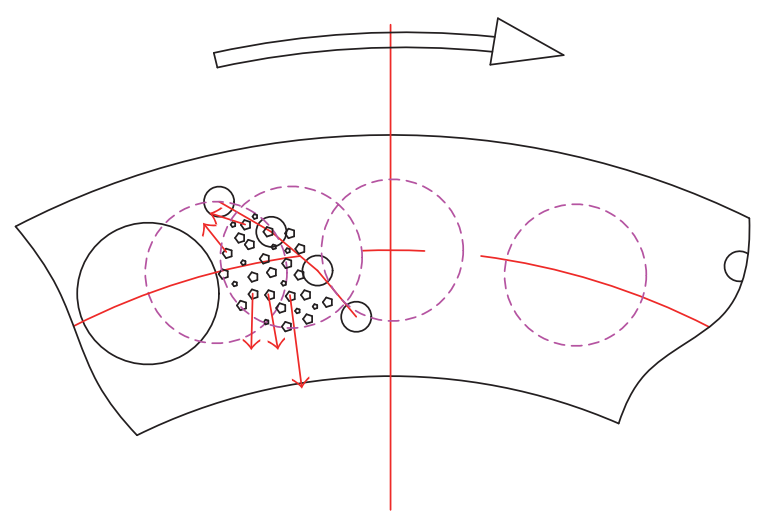

(b) Pin rotated clockwise

FIGURE 11: Influence mechanism of rotation direction on the tribological behavior of pit textured surface.

due to the microhydrodynamic lubrication effect of pits, the textured surface can offer the better tribological outcomes than its performance in dry wear conditions $[25,26]$.

\section{Conclusions}

Through theoretical analysis, numerical simulation, and experimental analysis, the influence of relative rotation direction between a textured disc and a pin on the tribological behavior of a pin-on-disc system with curve distributed pit textured surfaces was investigated. The influence mechanism was also proposed and discussed and the following conclusions could be drawn.

(1) The relative rotation direction between the textured disc and the pin has a significant influence on the thermal behavior of pit textured surfaces, which can be proved, whether from the temperature curves/contours, or from the von Mises stress curves/contours. When the pin rotated in an anticlockwise direction, the average temperature and von Mises stress were both lower than those when the pin rotated clockwise.

(2) Independent of rotation direction, there was an evident uneven temperature phenomenon on the texture surface, called "temperature crawling," which might be induced by "stick-slip" behavior during friction.

(3) When the pin rotated in an anticlockwise direction, the friction coefficient and wear loss were lower than those observed as the pin rotated in the contradirection. All the experimental results were in good agreement with the results of simulation, indicating that the anticlockwise rotation direction of the pin was a better choice under the conditions of this work.

The influence of rotating speed on the tribological behavior of pit textured surfaces will be further researched in the future, for higher rotating speeds may reduce the temperature difference when the pin rotated anticlockwise or clockwise. The tribological behavior of other typical texture units, for example, "groove" and "grid," as well as the prediction of friction and mass loss, will also be reported in the future, based on "element birth and death" technique and other FEA methods.

\section{Conflicts of Interest}

The authors declare that they have no conflicts of interest.

\section{Acknowledgments}

The research is supported by China Scholarship Council and the Qualified Personnel Foundation of Taiyuan University of Technology (TYUT-RC201426a) as well as the 2014 Shanxi Coal-Based Key Scientific \& Technological Projects (MJ201406). The authors would like to acknowledge Shanxi Key Laboratory of Fully Mechanized Coal Mining Equipment, China.

\section{References}

[1] H. Zhou, X. Tong, Z. Zhang, X. Li, and L. Ren, “The thermal fatigue resistance of cast iron with biomimetic non-smooth surface processed by laser with different parameters," Materials Science and Engineering A, vol. 428, no. 1-2, pp. 141-147, 2006.

[2] W. Shizhu and P. Huang, Principles of Tribology, Tsinghua University Press, Beijing, China, 2008.

[3] C. J. Evans and J. B. Bryan, "'Structured", "textured" or "engineered" surfaces," CIRP Annals - Manufacturing Technology, vol. 48, no. 2, pp. 541-556, 1999.

[4] X. Shi and T. Ni, "Effects of groove textures on fully lubricated sliding with cavitation," Tribology International, vol. 44, no. 12, pp. 2022-2028, 2011.

[5] T. Ibatan, M. S. Uddin, and M. A. K. Chowdhury, "Recent development on surface texturing in enhancing tribological performance of bearing sliders," Surface and Coatings Technology, vol. 272, pp. 102-120, 2015.

[6] C. Meng, H. Zhou, Y. Zhou et al., "Influence of different temperatures on the thermal fatigue behavior and thermal stability of hot-work tool steel processed by a biomimetic couple laser technique," Optics and Laser Technology, vol. 57, pp. 57-65, 2014.

[7] K. W. Liew, C. K. Kok, and M. N. Ervina Efzan, "Effect of EDM dimple geometry on friction reduction under boundary and mixed lubrication," Tribology International, vol. 101, pp. 19, 2016. 
[8] S. Schreck and K.-H. Z. Gahr, "Laser-assisted structuring of ceramic and steel surfaces for improving tribological properties," Applied Surface Science, vol. 247, no. 1-4, pp. 616-622, 2005.

[9] H. Zhiwu, R. Luquan, and L. Zubin, "Investigation on anti-wear ability of bionic nonsmooth surfaces made by laser texturing," Tribology, vol. 24, no. 4, pp. 289-293, 2004 (Chinese).

[10] D. Baoqing, R. Luquan, Suyan et al., "Tribological study on bionically unsmoothed surface modeling piston-cylinder friction pair," Journal of Jilin University (Engineering and Technology Edition), vol. 34, no. 1, pp. 79-84, 2004 (Chinese).

[11] Y. Kligerman, I. Etsion, and A. Shinkarenko, "Improving tribological performance of piston rings by partial surface texturing," Journal of Tribology, vol. 127, no. 3, pp. 632-638, 2005.

[12] X. Wang, W. Liu, F. Zhou, and D. Zhu, "Preliminary investigation of the effect of dimple size on friction in line contacts," Tribology International, vol. 42, no. 7, pp. 1118-1123, 2009.

[13] Liyang, D. Haishun, and W. Xiaolei, "Experiment on friction and wear performance of textured port plate pair," Journal of Aerospace Power, vol. 29, no. 7, pp. 1591-1596, 2014 (Chinese).

[14] P. Andersson, J. Koskinen, S. Varjus et al., "Microlubrication effect by laser-textured steel surfaces," Wear, vol. 262, no. 3-4, pp. 369-379, 2007.

[15] L. Junling, C. Ping, S. Tianmin, and X. Xin, "The effect of gourdshaped surface texture on tribological performance of stainless steel," Tribology, vol. 36, no. 2, pp. 207-214, 2016.

[16] H. Zhang, T. Zhou, H. Zhou, Z. Chen, W. Yang, and L. Ren, "Effects of different bionic units coupling on the sliding wear of gray cast iron," Surface and Coatings Technology, vol. 309, pp. 96-105, 2017.

[17] H. Zhang, P. Zhang, Q. Sui, K. Zhao, H. Zhou, and L. Ren, "Influence of multiple bionic unit coupling on sliding wear of laser-processed gray cast iron," Journal of Materials Engineering and Performance, vol. 26, no. 4, pp. 1614-1625, 2017.

[18] E. Gualtieri, A. Borghi, L. Calabri, N. Pugno, and S. Valeri, "Increasing nanohardness and reducing friction of nitride steel by laser surface texturing," Tribology International, vol. 42, no. 5, pp. 699-705, 2009.

[19] W. Hongtao and Z. Hua, "Effect of cylindrical micro-pit's distribution form on tribology properties of textured surface," Tribology, vol. 34, no. 4, pp. 414-419, 2014 (Chinese).

[20] S. Yuan, W. Huang, and X. Wang, "Orientation effects of microgrooves on sliding surfaces," Tribology International, vol. 44, no. 9, pp. 1047-1054, 2011.

[21] H. L. Costa and I. M. Hutchings, "Effects of die surface patterning on lubrication in strip drawing," Journal of Materials Processing Technology, vol. 209, no. 3, pp. 1175-1180, 2009.

[22] U. Pettersson and S. Jacobson, "Friction and wear properties of micro textured DLC coated surfaces in boundary lubricated sliding," Tribology Letters, vol. 17, no. 3, pp. 553-559, 2004.

[23] N. Kawasegi, H. Sugimori, H. Morimoto, N. Morita, and I. Hori, "Development of cutting tools with microscale and nanoscale textures to improve frictional behavior," Precision Engineering, vol. 33, no. 3, pp. 248-254, 2009.

[24] S. Shaoni, X. Liyang, and Z. Yichen, "Finite element analysis on friction and wear properties of non-smooth surface brake disc," Journal of Northeastern University (Natural Science), vol. 35, no. 11, pp. 1597-1601, 2014 (Chinese).

[25] J. Echávarri Otero, E. de la Guerra Ochoa, I. Bellón Vallinot, and E. Chacón Tanarro, "Optimising the design of textured surfaces for reducing lubricated friction coefficient," Lubrication Science, vol. 29, no. 3, pp. 183-199, 2017.
[26] C. Gachot, A. Rosenkranz, S. M. Hsu, and H. L. Costa, "A critical assessment of surface texturing for friction and wear improvement," Wear, vol. 372-373, pp. 21-41, 2017. 


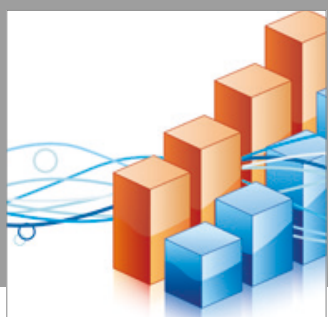

Advances in

Operations Research

vatersals

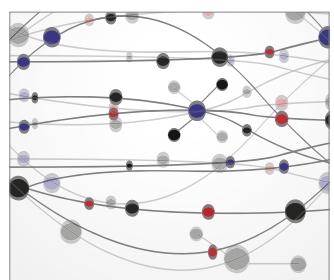

\section{The Scientific} World Journal
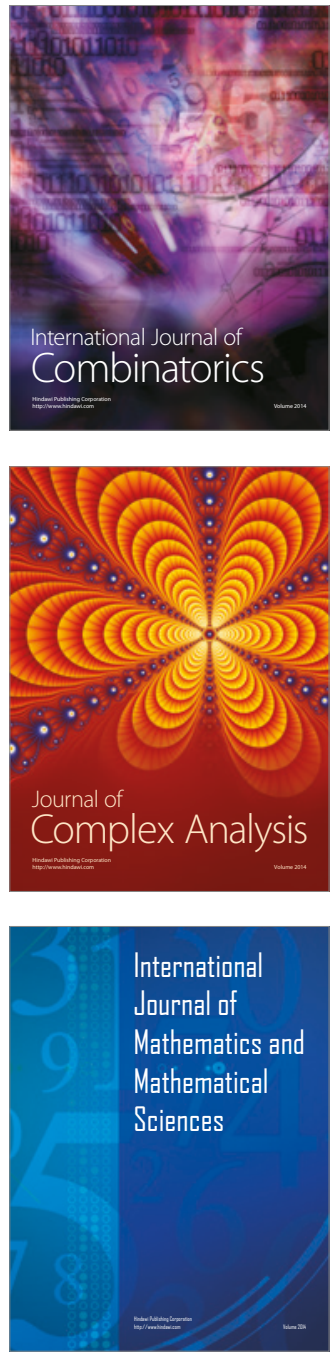
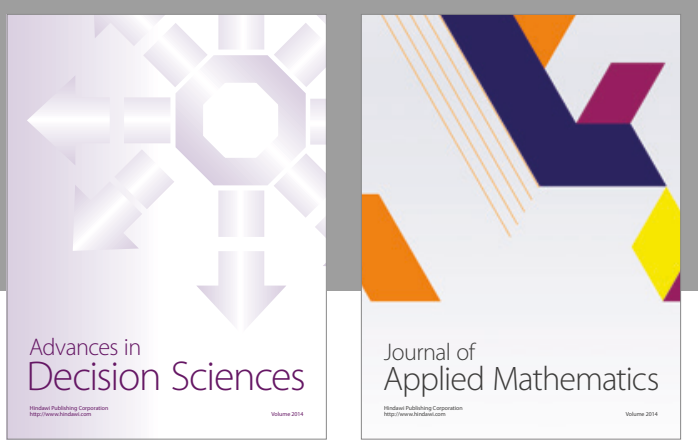

Algebra

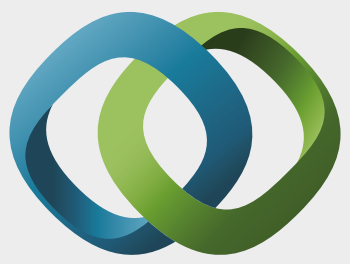

\section{Hindawi}

Submit your manuscripts at

https://www.hindawi.com
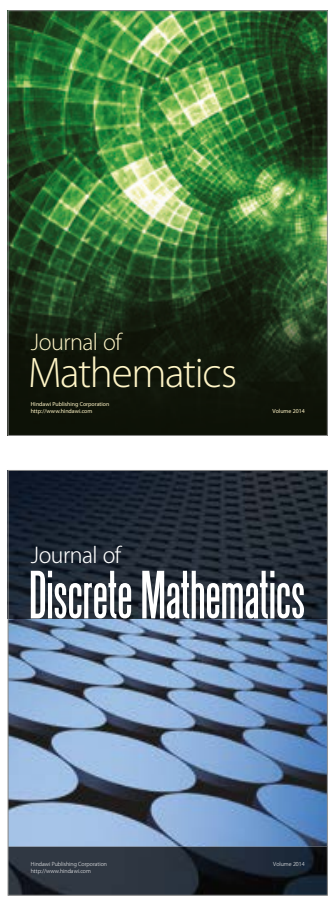

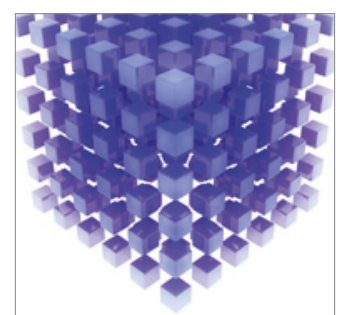

Mathematical Problems in Engineering
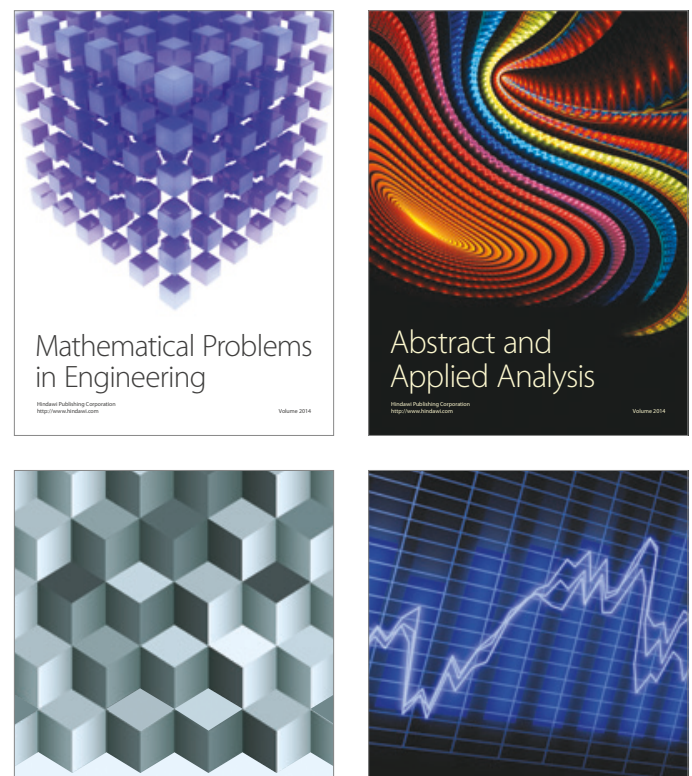

Journal of

Function Spaces

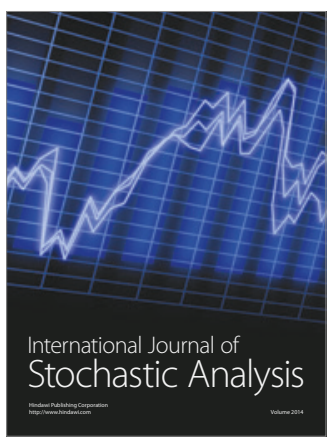

Probability and Statistics
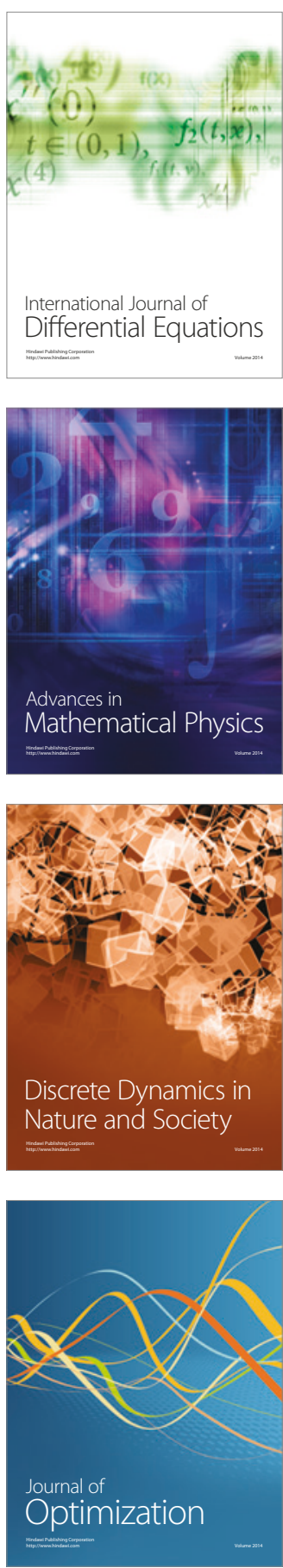